\title{
PREVALÊNCIA, INTENSIDADE E DESCONFORTO DA SEDE NO PACIENTE CIRÚRGICO NO PÓS-OPERATÓRIO IMEDIATO
}

\section{Prevalence, intensity and discomfort of thirst in surgical patients in the immediate post-operative period}

\section{Prevalencia, intensidad y desconforto de la sed en el paciente quirúrgico en el post-operatorio inmediato}

\author{
Leonel Alves do Nascimento ${ }^{*}$ (D), Thammy Gonçalves Nakaya ${ }^{2}$ (D), Marilia Ferrari Conchon ${ }^{3}$ (D), Aline Korki Arrabal Garcia2 ${ }^{2}$, \\ Isadora Pierotti ${ }^{2}$ (D) , Viviane Moreira Serato ${ }^{4}$, Lígia Fahl Fonseca ${ }^{7}$ (D)
}

RESUMO: Objetivo: Avaliar a prevalência, a intensidade e o desconforto da sede no período pós-operatório imediato. Método: Estudo epidemiológico, transversal, descritivo, quantitativo, realizado em um hospital universitário do Sul do Brasil, de agosto a setembro de 2012. A amostra foi composta de 386 pacientes em recuperação anestésica de cirurgias eletivas e de urgência. Foi utilizado um questionário semiestruturado composto de dados demográficos, clínicos e variáveis relacionadas à sede. Resultados: A prevalência de sede foi de $78 \%$ (303 pacientes), com intensidade média de 6,94 (desvio padrão — DP=2,2) e queixa espontânea de sede em 38,3\% dos casos (116 pacientes). Os desconfortos relatados foram: boca seca, procura por água, hipossalivação, garganta seca, lábios ressecados, língua seca e vontade de deglutir. Todos os desconfortos apresentaram correlação de Pearson positiva em relação à presença de sede. Conclusão: A sede no pós-operatório imediato é intensa, prevalente e com sinais periféricos desconfortáveis. Essas evidências fundamentam a necessidade da identificação, da mensuração, da avaliação e do tratamento do sintoma sede de forma intencional neste período. Palavras-chave: Sede. Cuidados de enfermagem. Enfermagem perioperatória. Prevalência.

ABSTRACT: Objective: To assess the prevalence, intensity and discomfort of thirst in the immediate postoperative period. Method: This is a cross-sectional, descriptive, quantitative epidemiological study conducted in a university hospital in the South of Brazil, from August to September 2012 . The sample consisted of 386 patients in anesthesia recovery from elective and emergency surgeries. A semi-structured questionnaire was used, consisting of demographic, clinical, and thirst-related variables. Results: The prevalence of thirst was $78 \%$ (303 patients), with an average intensity of 6.94 (standard deviation $-S D=2.2$ ) and spontaneous complaint of thirst in $38.3 \%$ of the cases (116 patients). The discomforts reported were: dry mouth, search for water, hyposalivation, dry throat, dry lips, dry tongue and willingness to swallow. All discomforts presented a positive Pearson correlation as to the presence of thirst. Conclusion: Immediate postoperative thirst is intense, prevalent and with uncomfortable peripheral signs. These facts substantiate the need to intentionally identify, measure, evaluate and treat thirst in this period. Keywords: Thirst. Nursing care. Perioperative nursing. Prevalence.

RESUMEN: Objetivo: Evaluar la prevalencia, la intensidad y el malestar de la sed en el período postoperatorio inmediato. Método: Estudio epidemiológico, transversal, descriptivo, cuantitativo, realizado em un hospital universitario del sur de Brasil, de agosto a septiembre de 2012. La muestra fue compuesta de 386 pacientes en recuperación anestésica de cirugías electivas y de urgencia. Se utilizó un cuestionario semiestructurado compuesto de datos demográficos, clínicos y variables relacionados con la sed. Resultados: La prevalencia de sed fue de 78\%(303 pacientes), con una intensidad media de 6,94(desviación estándaro standard deviation-SD=2,2) y queja espontánea de sed en el 38,3\% de los casos (116 paciente). Las incomodidades reportadas fueron: boca seca, busca por agua, hiposalivación, garganta seca, labios secos, lengua seca y voluntad de deglutir. Todas las incomodidades presentaron correlación de Pearson positiva en relación a la presencia de sed. Conclusión: La sed en el postoperatorio inmediato es intensa, prevalente y con señales periféricas incómodas. Estas evidencias fundamentan la necesidad de identificar, medir, evaluary tratar el síntoma de forma intencional en este período. Palabras clave: Sed. Atención de enfermería. Enfermería perioperatoria. Prevalencia.

'Enfermeiro; doutorando em Enfermagem pela Universidade Estadual de Londrina (UEL) - Londrina (PR), Brasil.

'Enfermeira; doutoranda em Enfermagem pela UEL - Londrina (PR), Brasil.

${ }^{3}$ Enfermeira; doutora em Enfermagem pela Universidade de São Paulo (USP). Membro do Grupo de Estudo e Pesquisa da Sede, da UEL - Londrina (PR), Brasil.

"Enfermeira; mestre em Enfermagem pela UEL. Membro do Grupo de Estudo e Pesquisa da Sede - Londrina (PR), Brasil.

'Enfermeira; doutora em Enfermagem, Programa de Saúde do Adulto pela USP. Professora associada do Departamento de Enfermagem da UEL - Londrina (PR), Brasil.

*Autor correspondente: leonel_lan@hotmail.com

Recebido: 17/08/2018 - Aprovado: 23/03/2019

DOI: 10.5327/Z1414-4425201900020006 


\section{INTRODUÇÃO}

A sede é uma necessidade humana vital derivada de um complexo sistema de sinalização neuro-hormonal que regula o equilíbrio hidroeletrolítico. Por ser um desconforto subjetivo, é caracterizada como um sintoma e leva a uma intensa motivação, desde a busca até o consumo de água, de forma que não pode ser ignorada ${ }^{1-3}$.

Por ser um sintoma multifatorial, a sede pode ser mais bem compreendida se analisados os fatores individuais que interferem em seu surgimento e na percepção que se tem dela. Cada paciente vivencia, avalia e enfrenta a sede de maneira particular, especialmente quando associada aos estímulos estressantes advindos do período perioperatório ${ }^{4,5}$.

A percepção da sede e o desejo de ingerir líquidos para atingir a saciedade são processos fisiológicos condicionados em todos os seres humanos ${ }^{6,7}$. O paciente cirúrgico, especialmente, tem sua sede potencializada por fatores como jejum pré-operatório, medicações utilizadas e perda sanguínea no intraoperatório. Esses estímulos resultam em comportamentos negativos, tais como estresse, ansiedade, irritabilidade e desespero, que intensificam o desconforto da sede no período perioperatório ${ }^{8-10}$.

Esse desconforto exibe elevada prevalência, chegando a patamares de $83,7^{11}$ a $88,6 \%{ }^{12}$ no paciente cirúrgico no período pós-operatório imediato (POI). Além disso, estudos apontam que a sede apresenta alta intensidade $\left(6,10^{13}, 8,17^{11}\right.$ e $\left.8,70^{14}\right)$ quando mensurada por escala verbal numérica $(\mathrm{EVN})$, que varia de zero a dez, sendo zero nenhuma sede e dez a sede mais intensa já sentida. O desconforto da sede também pode ser expresso pelos próprios pacientes por um conjunto de sintomas periféricos, também denominados atributos da sede, como lábios ressecados, boca e garganta secas, língua e saliva grossas, gosto ruim ou amargo na boca, e pela vontade de beber água ${ }^{2,3,15-17}$.

Ainda que tradicionalmente não se atente para a ocorrência do sintoma sede como fator relevante para avaliação, mensuração e tratamento durante a permanência do paciente na Sala de Recuperação Anestésica (SRA), deve-se atentar para esse desconforto, considerando sua complexidade e o fato de ser uma das experiências humanas mais estressoras e impetuosas no $\mathrm{POI}^{3,10}$.

\section{OBJETIVO}

Avaliar a prevalência, a intensidade e o desconforto da sede em pacientes cirúrgicos no período POI.

\section{MÉTODO}

Trata-se de um estudo epidemiológico, de corte transversal, descritivo e de abordagem quantitativa, realizado na SRA de um hospital-escola público e de nível terciário no Sul do Brasil. A instituição possui 313 leitos, todos à disposição do Sistema Único de Saúde (SUS). O centro cirúrgico (CC) conta com sete salas cirúrgicas $(\mathrm{SO})$ e realiza, em média, 640 cirurgias mensais.

A população foi constituída de pacientes adultos e idosos de ambos os sexos, submetidos a cirurgias eletivas e de urgência, que se encontravam no POI, na SRA. Os critérios de inclusão foram: apresentar idade entre 18 e 65 anos e relatar sede espontaneamente ou quando questionado. Foram excluídos do estudo os pacientes que receberam alta da SO diretamente para a Unidade de Terapia Intensiva (UTI), Unidades de Internação ou domicílio, além de pacientes que apresentaram desorientação quanto ao tempo e ao espaço e com comprometimento na comunicação verbal.

A amostra foi constituída de 386 participantes, considerando nível de confiança de $95 \%$ e erro amostral de $5 \%$. A coleta de dados se deu no período de segunda a sexta-feira, das 7 às 19 horas, nos meses de agosto e setembro de 2012, e foi realizada por acadêmicos do curso de enfermagem e residentes de enfermagem perioperatória. Os acadêmicos foram capacitados por meio de treinamento realizado pelo pesquisador responsável do estudo, com o objetivo de manter o rigor metodológico e o cumprimento dos aspectos éticos durante a coleta.

O roteiro de coleta de dados contemplou informações demográficas, clínicas e variáveis relacionadas à sede, tais como presença de sede, queixa espontânea, características da sede e intensidade do desconforto avaliada pela EVN, que variou de 0 a 10 , sendo 0 nenhum desconforto e 10 o desconforto mais intenso já sentido. Os desconfortos avaliados foram: lábios ressecados, boca seca, língua grossa, garganta seca, hipossalivação, vontade de deglutir e procura por água. Outras variáveis foram avaliadas para permitir a compreensão de fatores que podem caracterizar ou interferir no processo da sede, como tempo de jejum pré-operatório, intubação orotraqueal e tempo do procedimento cirúrgico.

A coleta de dados foi dividida em dois momentos: pré-operatório e pós-operatório. No primeiro momento, na SRA, todos os pacientes que atenderam aos critérios de inclusão foram abordados, orientados quanto aos objetivos da pesquisa e convidados a participar do estudo. Todos os 
participantes assinaram o Termo de Consentimento Livre e Esclarecido (TCLE). No segundo momento, após o procedimento anestésico-cirúrgico, aplicou-se, na SRA, o instrumento de coleta de dados.

Para avaliar o sintoma sede no POI, aguardou-se a verbalização espontânea do paciente até a alta da SRA. Quando ela não ocorria, o pesquisador questionava o paciente sobre a presença ou a ausência de sede. Em caso afirmativo, aplicava a EVN para avaliar a intensidade do sintoma, solicitando, em seguida, que citasse as características por ele percebidas por estar com sede.

Os dados foram digitados em planilha no Excel 2010, e a análise estatística foi realizada no programa Statistical Products and Service Solutions (SPSS), versão 20.0, considerando o nível de significância de $5 \%$. Para correlacionar a sede e as características referidas, utilizou-se o Teste $\chi^{2}$ de Pearson.

O estudo seguiu as condições estabelecidas na Resolução $n^{\circ}$ 466/2012 do Conselho Nacional de Saúde (CNS) e foi aprovado pelo Comitê de Ética da Universidade Estadual de Londrina (CEP-UEL) (CAAE: 02299412.6.0000.5231 - CEP $11037 / 2012$ ). Todos os pacientes foram convidados e, após $\mathrm{o}$ aceite, assinaram o TCLE.

\section{RESULTADOS}

Entre os 386 participantes, predominou o sexo masculino, com 217 (56\%) pacientes. A média de idade foi de 42,3 anos (12-89 desvio padrão - $\mathrm{DP}=18,3)$. Em relação ao tempo de jejum pré-operatório, a média em horas de jejum absoluto foi de 17,53 (6,15-35,50 h DP=4,54 h), e predominou um tempo de jejum superior a 16 horas em 241 (62\%) pacientes. A clínica cirúrgica com maior frequência foi a ginecologia e obstetrícia, com $109(28,2 \%)$ procedimentos realizados, seguida pela ortopedia, com $71(18,4 \%)$.
A maioria foi classificada como ASA I (194 pacientes 50\%), seguindo-se ASA II (127 pacientes - 33\%), ASA III (60 pacientes - 16\%) e ASA IV (5 pacientes - 1\%). A técnica anestésica de bloqueio raquidiano foi a mais utilizada (199 pacientes - 51,6\%), seguida pela anestesia geral balanceada (121 pacientes - 31,3\%) e por outras técnicas (66 pacientes $-17 \%$ ).

Houve prevalência de sede no POI em 303 (78\%) pacientes. A intensidade média do sintoma foi de 6,94 (DP 2,2). A sede foi intensa (7-10) para 132 (34\%) participantes.

Quanto à avaliação da queixa de sede, $116(38,3 \%)$ pacientes se queixaram espontaneamente do desconforto, e 187 $(61,7 \%)$ referiram sede depois de questionados sobre o sintoma. O coeficiente de associação de $\chi^{2}$ de Pearson entre a presença de sede e os desconfortos foi estatisticamente significativo (Tabela 1).

Os desconfortos apresentados pelos pacientes e relacionados com a intensidade da sede estão citados na Figura 1.

Houve necessidade de intubação orotraqueal em 126 $(32,6 \%)$ sujeitos. Testes de associação de $\chi^{2}$ de Pearson entre a presença de sede e a intubação orotraqueal foram estatisticamente significativos $\left(\chi^{2}=7,11 \mathrm{p}=0,008\right.$ Coeficiente phi $(\mathrm{Fi})=0,136)$, denotando que, independentemente do total de intubados, há associação entre o procedimento e a presença de sede.

\section{DISCUSSÃO}

A relevância do estudo está na avaliação e na identificação de atributos da sede na SRA, pois não se encontram registros de sintoma tão prevalente e distressor no período de recuperação, revelando uma realidade oculta, de grande magnitude, negligenciada e que repercute em experiências

Tabela 1. Associação entre presença de sede e desconfortos apresentados pelos pacientes no pós-operatório imediato ( $\mathrm{n}=386$ ).

\begin{tabular}{|c|c|c|c|c|c|}
\hline Desconfortos & $\mathbf{n}$ & $\% *$ & $\mathbf{F i}^{* *}$ & $\mathbf{X}^{2 * *}$ & $\mathbf{p}^{* * *}$ \\
\hline Lábios ressecados & 87 & 22,5 & 0,282 & 30,76 & 0,000 \\
\hline Boca seca & 267 & 69,2 & 0,784 & 237,24 & 0,000 \\
\hline Língua grossa & 54 & 14,0 & 0,211 & 17,19 & 0,000 \\
\hline Garganta seca & 91 & 23,6 & 0,291 & 32,61 & 0,000 \\
\hline Hipossalivação & 105 & 27,2 & 0,320 & 39,51 & 0,000 \\
\hline Vontade de deglutir & 37 & 9,6 & 0,170 & 11,21 & 0,001 \\
\hline Procura por água & 141 & 36,5 & 0,397 & 60,85 & 0,000 \\
\hline Não apresentou desconforto & 04 & 1,0 & 0,054 & 1,10 & 0,293 \\
\hline
\end{tabular}

*Pode-se relatar mais de um desconforto; **Fi: Coeficiente phi/Graus de liberdade :1; ***p>0,001. 
pós-cirúrgicas negativas, quando não identificadas e tratadas de forma segura e humanizada.

A prevalência da sede pós-operatória neste estudo foi de $78 \%$, demonstrando a relevância e a expressividade que esse sintoma tem na vivência do paciente no POI. Outros estudos corroboram esse achado, ao demonstrarem prevalências também elevadas, entre $75^{13}, 83,7^{11}$ e $88,6 \%{ }^{12}$ em adultos, e $88,5 \%{ }^{18}$ em crianças.

Os desconfortos relatados pelos participantes eram marcantes. Sabe-se que a gênese da sede e seus desconfortos no paciente cirúrgico são multifatoriais. A experiência cirúrgica e as reações emocionais, como estresse, medo e ansiedade, desencadeiam reações bioquímicas e hormonais para a manutenção da homeostase do organismo ${ }^{2,9}$. Essas respostas inibem a produção de saliva pelas glândulas salivares, causando ressecamento da mucosa oral e da região orofaríngea ${ }^{2,15,16}$, que os pacientes cirúrgicos relatam como boca seca.

Entre os desconfortos da sede, o mais relatado pelos pacientes é boca seca, o que revela quão incômodo este atributo pode se tornar. Desse modo, a percepção da sede não ocorre apenas pela presença consciente e pela intensidade da sede avaliada, mas também pela presença de sinais periféricos, como boca seca, lábios ressecados, língua e saliva grossas, garganta seca, gosto ruim na boca, halitose, e pela vontade de beber água ${ }^{2,16,17}$.

Já no período pré-operatório, pode-se identificar a presença de reações bioquímicas e hormonais advindas de uma confluência de fatores, entre eles a restrição hídrica e alimentar acima do preconizado pelas diretrizes das sociedades responsáveis, tais como a American Society of Anesthesiologists $(\text { ASA })^{19}$. Neste estudo, a média de horas de jejum absoluto pré-operatório foi de 17,53 , o que representa aumento exponencial do desconforto experienciado pelos pacientes, resultado consoante com uma pesquisa em que os pacientes permaneceram, em média, $16,5 \mathrm{~h}$ de jejum pré-operatório ${ }^{20}$.

Em um estudo para desenvolver e validar uma escala de desconforto da sede perioperatória, sete atributos foram elencados como os mais representativos do desconforto da sede: boca seca, lábios ressecados, língua grossa, saliva grossa, garganta seca, gosto ruim na boca e vontade de beber água ${ }^{17}$. A maioria desses atributos relaciona-se aos relatados no presente estudo, demonstrando que os desconfortos expressos pelos pacientes são significativos e devem ser ouvidos, considerados e tratados com intencionalidade.

No que se refere ao relato, tanto da sede como dos desconfortos a ela relacionados, observou-se, neste estudo, que $61,7 \%$ dos participantes, mesmo vivenciando a sede de maneira intensa, não a verbalizaram. Resultado semelhante descreveu que $88 \%$ dos pacientes não verbalizaram, de forma espontânea, o sintoma ${ }^{12}$.

O silêncio que permeia o paciente cirúrgico é reflexo da subvalorização da sede pelos profissionais de saúde. Desconfortos como náuseas, vômitos e dor são identificados e tratados prontamente, enquanto a sede perioperatória, mesmo com alta prevalência, é pouco questionada e tratada ${ }^{21}$. O silêncio talvez seja indício de "não esquecimento" de um sofrimento ${ }^{22}$ que contribui para experiências hospitalares angustiantes ${ }^{10}$.

Essa realidade inquietante foi alvo de um estudo para explorar a percepção do paciente cirúrgico em relação aos motivos que o levam a não verbalizar sua sede espontaneamente

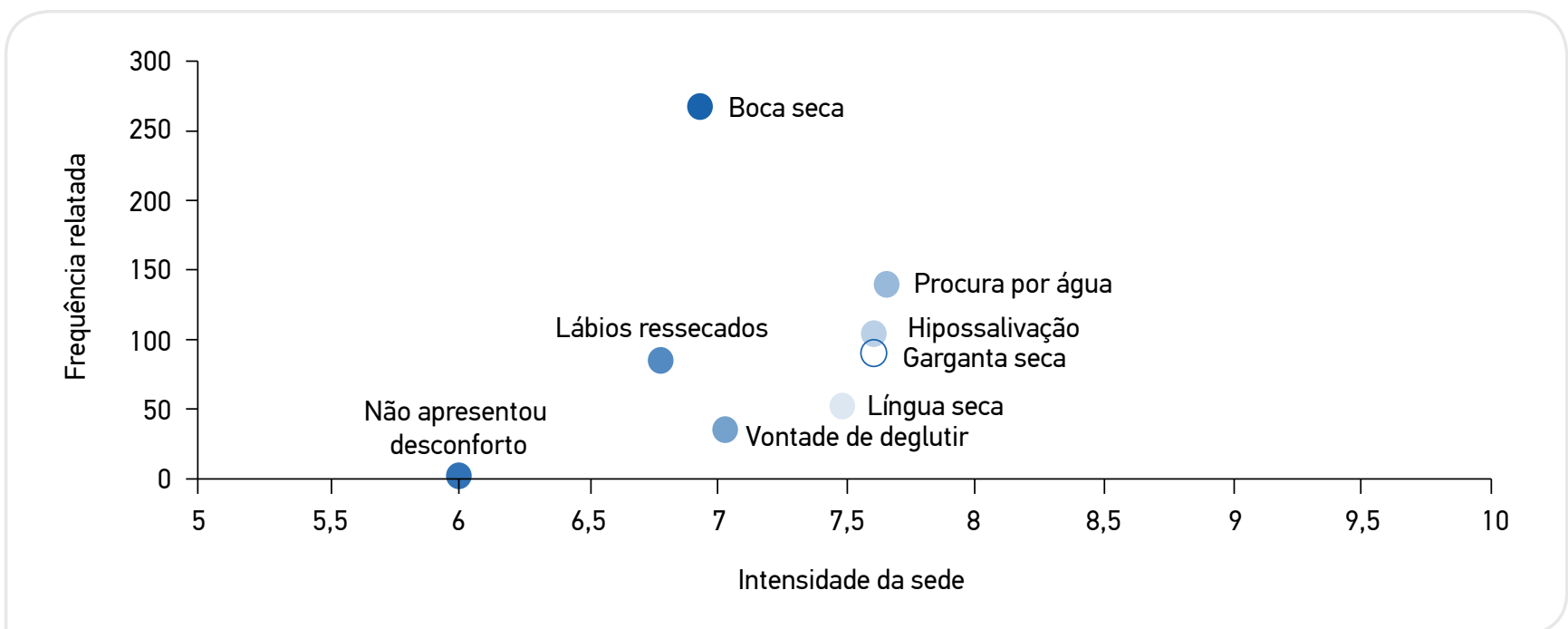

Figura 1. Frequência dos desconfortos citados em relação à intensidade média de sede em pacientes no pós-operatório imediato ( $\mathrm{n}=386$ ). 
aos profissionais de saúde. O paciente teme exteriorizar suas sensações em relação à sede por entender que é um sintoma inerente ao ato anestésico-cirúrgico, acatando quando profissionais reforçam a manutenção do jejum absoluto e a necessidade de suportar esse desconforto. O paciente considera papel da equipe perguntar a ele se sente sede; se a equipe não o questiona, ele silencia por temor de se posicionar e não expõe o desconforto imposto e limitante ${ }^{23}$. O mito de que a sede no perioperatório é um preço a ser pago para manter a vida está enraizada na cultura dos sistemas de saúde, compartilhada pelos profissionais e já também pelos pacientes ${ }^{3}$.

Tais evidências corroboram os resultados do presente estudo, revelando a necessidade de a equipe cirúrgica se apropriar dos saberes científicos a ponto de mudar essa realidade. Sabe-se que o paciente cirúrgico pertence a um grupo com alto risco de desenvolver sede, e já existem, no cenário científico, movimentos que justificam e descrevem a importância do desenvolvimento de estratégias de identificação, mensuração, avaliação da segurança, administração de métodos de alívio e registro do sintoma sede pautados na literatura, instrumentalizando os profissionais no cuidado do paciente cirúrgico com sede $\mathrm{e}^{3,17,24}$.
A valorização da sede perioperatória pelos profissionais de saúde e o olhar intencional para esse sintoma em sua prática clínica, além de transformarem positivamente a experiência do paciente cirúrgico, atendem a uma necessidade humana vital e acrescentam humanização e qualidade ao atendimento cirúrgico.

\section{CONCLUSÃO}

A sede no paciente cirúrgico no POI durante o período de recuperação anestésica se mostrou prevalente, intensa e desconfortável. Entre os atributos relacionados ao desconforto do sintoma, o mais experienciado foi boca seca. Esses dados corroboram outros estudos, apontando que o paciente cirúrgico apresenta alto risco de desenvolver sede, sintoma distressor que deve ser identificado, avaliado, mensurado e tratado de forma intencional, com o objetivo de melhoria da qualidade do cuidado perioperatório. Os resultados deste estudo apontam, portanto, para a necessidade de novas investigações e adoção de medidas para minorar o desconforto do paciente na recuperação anestésica.

\section{REFERÊNCIAS}

1. Antunes-Rodrigues J, Elias LLK, Castro M, De Luca Júnior LA, Vivas LM, Menani JV. Controleneuroendócrino do balanço hidroeletrolítico. In: Aires MM, editor. Fisiologia. $4^{\mathrm{a}}$ ed. Rio de Janeiro: Guanabara Koogan; 2012. p. 1181-98.

2. Arai S, Stotts N, Puntillo K. Thirst in critically ill patients: from physiology to sensation. Am J Crit Care [Internet]. 2013 [acessado em 25 maio 2018];22(4):328-35. Disponível em: https://www.ncbi. nlm.nih.gov/pubmed/23817822. https://doi.org/10.4037/ajcc2013533

3. Conchon MF, Nascimento LA, Fonseca LF, Aroni P. Perioperative thirst: an analysis from the perspective of the Symptom Management Theory. Rev Esc Enferm USP. 2015;49(1):120-6. http://dx.doi.org/10.1590/ S0080-623420150000100016

4. Dodd M, Janson S, Facione N, Faucett J, Froelicher ES, Humphreys J, et al. Advancing the science of symptom management. J Adv Nurs. 2001;33(5):668-76.

5. Humphreys J, Lee KA, Carrieri-Kohlman V, Puntillo K, Faucett J, Janson S, et al. Theory of Symptom Management. In: Smith MJ, Liehr PR, editors. Middle range theory for nursing. $2^{\mathrm{a}}$ ed. Nova York: Springer; 2008. p. 145-58.

6. Leiper JB. Thirst. In: Caballero B, Allen L, Prentice A, editores. Encyclopedia of human nutrition. 2a ed. Oxford: Elsevier; 2005.
7. Zimmerman CA, Leib DE, Knight ZA. Neural circuits underlying thirst and fluid homeostasis. Nat Rev Neurosci. [Internet]. 2017 [acessado em $1^{\circ}$ jun. 2018];18:459-69. Disponível em: https:// www.nature.com/articles/nrn.2017.71. https://doi.org/10.1038/ nrn.2017.71

8. Dessotte CAM, Rodrigues HF, Furuya RK, Rossi LA, Dantas RAS. Stressors perceived by patients in the immediate postoperative of cardiac surgery. Rev Bras Enferm. 2016;69(4):694-703. http://dx.doi. org/10.1590/0034-7167.2016690418i

9. Medeiros VCC, Peniche ACG. The influence of anxiety in coping strategies used during the pre-operative period. Rev EsC Enferm USP. 2006;40(1):86-92. http://dx.doi.org/10.1590/ S0080-62342006000100012

10. Silva LCJR, Aroni P, Fonseca LF. Tenho sede! Vivência do paciente cirúrgico no período perioperatório. Rev SOBECC. 2016;21(2):75-81. https://doi.org/10.5327/Z1414-4425201600020003

11. Conchon MF, Fonseca LF. Efficacy of an ice popsicle on thirst management in the immediate postoperative period: a randomized clinical trial. J Perianesth Nurs [Internet]. 2018 [acessado em 15 jul. 2018];33(2):153-61. Disponível em: https://doi.org/10.1016/j. jopan.2016.03.009 
12. Pierotti I, Fracarolli IFL, Fonseca LF, Aroni P. Avaliação da intensidade e desconforto da sede perioperatória. Rev Esc Anna Nery [Internet]. 2018 [acessado em 11 jun. 2018];22(3):e20170375. Disponível em: http:// www.scielo.br/pdf/ean/v22n3/pt_1414-8145-ean-22-03-e20170375. pdf. https://doi.org/10.1590/2177-9465-EAN-2017-0375

13. Aroni P, Nascimento LA, Fonseca LF. Assessment strategies for the management of thirst in the post-anesthetic recovery room. Acta Paul Enferm. 2012;25(4):530-6. http://dx.doi.org/10.1590/ S0103-21002012000400008

14. Cho EA, Kim KH, Park JY. Effects of frozen gauze with normal saline and ice on thirst and oral condition of laparoscopic cholecystectomy patients: pilot study. J Korean Acad Nurs [Internet]. 2010 [acessado em 2 jun. 2018];40(5):714-23. Disponivel em: https://doi.org/10.4040/ jkan.2010.40.5.714

15. Gebremedhn EG, Nagaratnam VB. Audit on perioperative fasting of elective surgical patients in an African academic medical center. World J Surgery [Internet]. 2014 [acessado em 9 jun. 2018];38(9):2200-4. Disponível em: https://doi.org/10.1007/s00268-014-2582-3

16. Landström M, Rehn IM, Frisman GH. Perceptions of registered and enrolled nurses on thirst in mechanically ventilated adult patients in intensive care units: a phenomenographic study. Intensive Crit Care Nurs [Internet]. 2009 [acessado em 13 jun. 2018];25(3):133-9. Disponivel em: https://doi.org/10.1016/j.iccn.2009.03.001

17. Martins PR, Fonseca LF, Rossetto EG. Developing and validating the Perioperative Thirst Discomfort Scale. Rev Esc Enferm USP. 2017;51:e03240. http://dx.doi.org/10.1590/s1980-220x2016029003240

18. Riviera AA. Prevalência e intensidade da sede infantil no pósoperatório imediato [dissertação]. Londrina: Universidade Estadual de Londrina; 2015 [acessado em 15 jun. 2018]. Disponível em: http:// www.bibliotecadigital.uel.br/document/?code=vtls000210646
19. Practice Guidelines for preoperative fasting and the use of pharmacologic agents to reduce the risk of pulmonary aspiration: application to healthy patients undergoing elective procedures: an updated report by the American Society of Anesthesiologists task force on preoperative fasting and the use of pharmacologic agents to reduce the risk of pulmonary aspiration. Anesthesiology [Internet]. 2017 [acessado em 21 jun. 2018];126(3):376-93. Disponível em: https://doi.org/10.1097/ALN.0000000000001452

20. Cestonaro T, Schieferdecker MEM, Thieme RD, Cardoso JN, Campos ACL. The reality of the surgical fasting time in the era of the ERAS protocol. Nutr Hosp [Internet]. 2014 [acessado em 2 jun. 2018];29(2):437-43. Disponível em: https://doi.org/10.3305/ nh.2014.29.2.7025

21. Pavani MM, Fonseca LF, Conchon MF. Sede no paciente cirúrgico: percepções da equipe de enfermagem nas unidades de internação. Rev Enferm UFPE [Internet]. 2016 [acessado em 18 jun. 2018];10(9):3352-60. Disponível em: https://periodicos.ufpe.br/ revistas/revistaenfermagem/article/download/11416/13201. https:// doi.org/10.5205/reuol.9571-83638-1-SM1009201621

22. Cardoso E, Cunha T. $O$ silêncio na comunicação. BOCC - Biblioteca On-line de Ciências e Comunicação. Portugal, Universidade Nova de Lisboa: LabCom, 2011.

23. Garcia AKA, Fonseca LF, Lodi CR. O silêncio que permeia a sede perioperatória: um estudo de caso. In: Anais do Encontro Internacional de Produção Científica da UNICESUMAR, 2017, Maringá [Internet]. 2017 [acessado em 14 jun. 2018]. Disponível em: http://sobecc. tmeventos.com.br/anais2017/pdfs/trabalho_2255.pdf

24. Nascimento LA, Fonseca LF, Santos C. Inter-rater reliability testing of the safety protocol for thirst management. J Perianesth Nurs [Internet]. 2018 [acessado em 5 ago. 2018];33(4):527-36. Disponível em: https://doi.org/10.1016/j.jopan.2016.07.008 